\title{
Quantitative Three Dimensional Echocardiography: Methodology, Validation, and Clinical Applications
}

\author{
Florence H. Sheehan M.D. ${ }^{1}$, Edward L. Bolson M.S. ${ }^{1}$, Roy W. Martin, Ph.D. *, \\ Gerard Bashein M.D. Ph. D.*, and John McDonald Ph.D. ${ }^{+}$
}

\begin{abstract}
Three dimensional (3D) ultrasound imaging provides the most accurate measurement of the volume of the heart's left ventricle. We have developed methods for acquiring and quantitatively analyzing $3 D$ echocardiograms to measure the size, shape, and function not only of the left ventricle but also the mitral annulus and other structures in the mitral apparatus. These methods provide a powerful tool to investigate structurefunction relationships, evaluate surgical procedures, and assess patient prognosis.
\end{abstract}

\section{INTRODUCTION}

Although multiple modalities are available clinically to image the heart, the elegant but complex structure of this organ is only now being revealed with the recent advent of 3D imaging techniques. Much of the research in 3D echocardiography to date has been devoted to volume rendering for visualization, e.g. of congenital heart defects. However quantitative analysis has the potential for much wider applications in adult cardiology.

Our research team has devoted 11 years to the development and validation of hardware and software for quantitative 3D echo. Acquiring a 3D echo study of a patient is performed using a magnetic field system to track the spatial location and orientation of each image. Our software allows us to display the images, trace the borders of heart structures, review the border tracings, reconstruct the left ventricle ( $\mathrm{LV})$, mitral valve, and associated structures in $3 \mathrm{D}$, and measure their dimensions, shape, and function.

This report presents the results of studies validating the accuracy of these techniques in phantoms and in heart specimens, and discusses applications of these techniques to clinical investigation.

\section{Methods}

Image Acquisition (Fig. 1) Imaging is performed using commercial uitrasound equipment. The 3D image set is acquired over four to six 6-10 sec periods of held end-expiration, depending on each patient's breath holding ability and image quality. The first two scans cover the parasternal long axis view and angulated short axis views of the LV. At the apical window, two or three separate scans are used to acquire the

\footnotetext{
'From the Depts. of Medicine/Cardiology (Box 356422, phone (206) 543-4535, fax (206) 685-9394), Anesthesiology", and Statistics ${ }^{+}$, University of Washington, Seattle, WA 98195
} 


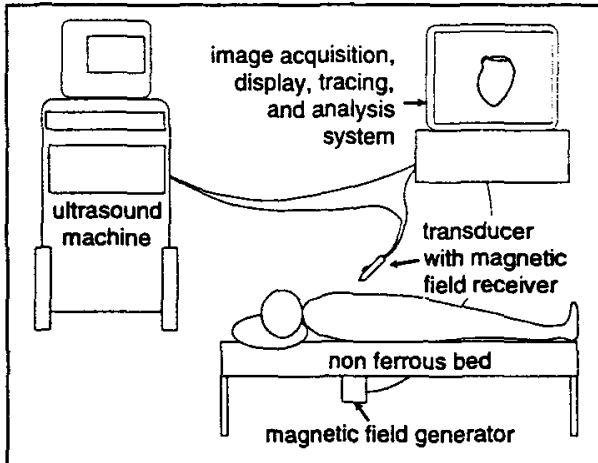

Figure 1. Schematic diagram of 30 echo imaging system

long axis images and complete a $180^{\circ}$ rotation. The sixth scan is performed if and where additional coverage is needed.

A magnetic field system (Flock of Birds $^{\mathrm{TM}}$ model 6D-FOB, Ascension Technology Corp., Burlington, VT) is used to track the ultrasound scanhead (1). The system consists of a magnetic field transmitter, a receiver, and electronic circuitry which interfaces with a computer. During imaging the transmitter is placed under the mattress and the receiver is attached to the ultrasound scanhead. Images are digitized using a framegrabber and registered with position data, hemodynamic parameters, and scanning parameters such as image depth.

Quantitative Analysis Techniques: The images are reviewed and those corresponding to end diastole and end systole are manually selected as the frames of maximum and minimum cavity area, respectively. The borders of the LV and of anatomic features are then manually traced in the selected images (Fig. 2). The border points are converted to $x, y, z$ coordinates using probe position and orientation data. The traced borders are reviewed immediately in 3D using a software interface (Advanced Visual Systems, Waltham MA). All images are traced using

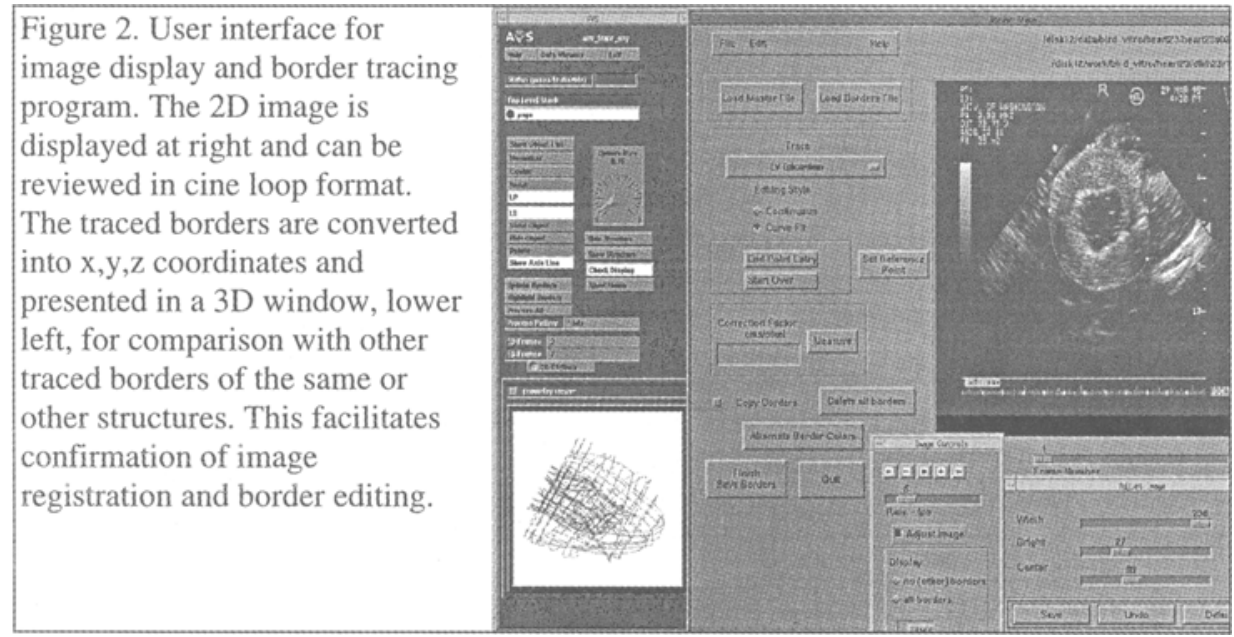


the leading-edge technique with the border passing through the middle of the bright part of the lateral echoes.

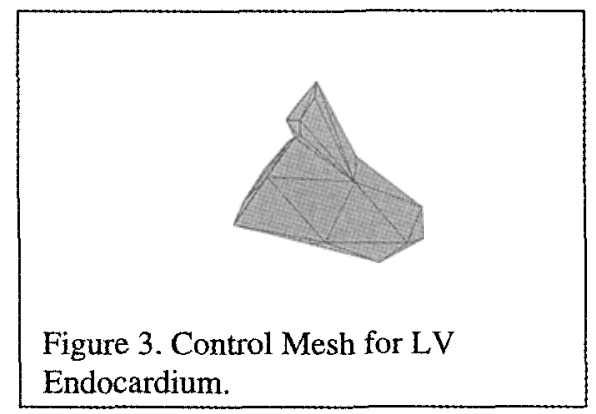

$3 D$ Reconstruction of the $L V$ endocardium and epicardium: A piecewise smooth subdivision surface is fit to $3 \mathrm{D}$ points sampled from the traced borders. This method was developed to avoid the inherent limitations of other methods which restricted their anatomical accuracy or application to different 3D image acquisition techniques. The piecewise smooth subdivision has been shown to accurately measure volume, and is the only method shown to reproduce LV shape with anatomical accuracy (1). This is essential for analysis of regional LV function and geometry. Triangulated control meshes are designed as models for the $\mathrm{LV}$ endocardium and epicardium (Fig. 3).

The control mesh is recursively subdivided to produce a mesh of 576 faces. Parts of the control mesh can be marked as sharp to allow for creases at the mitral annulus and outflow track and a sharp point at the apex in the final piecewise smooth subdivision surface. The control mesh is fit to the manually traced borders of the LV, valves, papillary muscles, apex, and other anatomic landmarks in a process that minimizes a penalized least-squares criterion which trades off fidelity to the borders against surface smoothness. The same mesh is used for both normal and diseased hearts (Fig.4).

\section{Figure 4.}

Reconstructions of the $\mathrm{LV}$

endocardium at end diastole (mesh) and end systole (solid) in a patient with cardiomyopathy (right) and a normal subject (left).

The piecewise smooth subdivision method is also used to reconstruct the surfaces of the papillary muscles (Fig. 5)

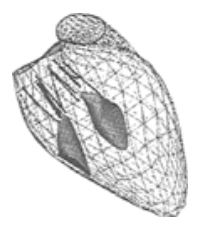

Figure 5. 3D reconstruction of the $\mathrm{LV}$, papillary muscles, and chordae tendineae.

and of the mitral valve leaflets (Fig. 6). 


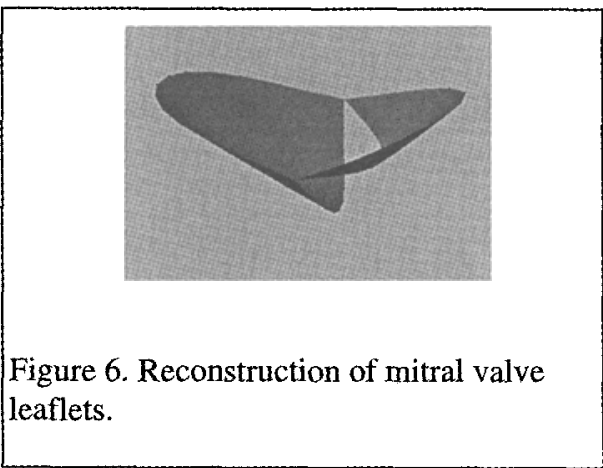

\section{Quantitative Analysis of Cardiac}

Parameters: LV volume is computed by summing the volumes of the tetrahedra formed by connecting a point inside the $\mathrm{LV}$ cavity with the vertices of each triangular face on the reconstructed 3D surface. The LV volumes determined at end diastole and end systole are used to calculate stroke volume, ejection fraction, and cardiac output, as well as the respective volume

\section{CenterSurface}

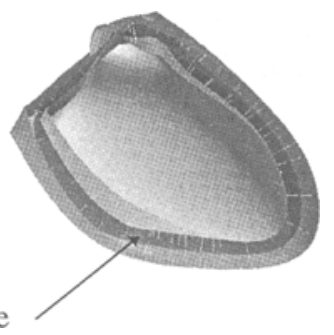

Figure 7. Cutaway view of reconstructions of the $\mathrm{LV}$ endocardium and epicardium and the CenterSurface ${ }^{\mathrm{TM}}$. indices. LV mass is calculated as the difference between the volumes of the LV endocardial and epicardial surface reconstructions, multiplied by the specific gravity of cardiac muscle.

To measure regional wall thickness, a medial surface (CenterSurface ${ }^{\mathrm{TM}}$ ) is constructed midway between the epicardial and endocardial surface reconstructions (2) (Fig. 7). Chords are drawn perpendicular to the CenterSurface ${ }^{\mathrm{TM}}$ and extended to their intersection with the endocardium and epicardium. The length of each chord is a measure of the local orthogonal wall thickness. Comparison of the wall thickness at end diastole and end systole at each triangular face of the LV surface provides a measure of wall thickening, a parameter of regional $\mathrm{LV}$ function.

To reconstruct the mitral valve annulus the traced points are ordered and then connected to form a polygon. A smooth curve is constructed using Fourier series approximations in $x, y$, and $z$ relative to path length along the polygon (3). The mitral annulus orifice area is determined from the least squares plane fitted to the annular data. Other dimensions such as annular height, circumference, eccentricity, as well as the distance from the LV apex to the annulus, the distance between high points on the annulus, the distance between low points on the annulus, and the magnitude of annular motion are analyzed from the reconstructed annulus. 


Table 1. In vitro Validation of Volume Measurement by 3D Echo
\begin{tabular}{|l|l|l|l|l|l|}
\hline Standard & Range, $\mathrm{ml}$ & $\mathrm{N}$ & Regression & $\mathrm{r}$ & SEE, $\mathrm{ml}$ \\
\hline beaker phantom & $48-151$ & 9 & $\mathrm{y}=1.05 \mathrm{x}-0.59$ & 0.995 & 3.5 \\
\hline balloon phantoms & $40.6-182.5$ & 12 & $\mathrm{y}=1.00 \mathrm{x}-0.6$ & 1.000 & 1.3 \\
\hline in vitro hearts & $30.2-104.1$ & 5 & $\mathrm{y}=1.02 \mathrm{x}-1.3$ & 1.000 & 0.4 \\
\hline Doppler stroke volume & $67.2-117.8$ & 5 & $\mathrm{y}=1.18 \mathrm{x}-17.9$ & 0.990 & 2.8 \\
\hline
\end{tabular}

\begin{tabular}{|c|c|c|c|c|c|}
\hline Standard & Range,gm & $\mathrm{N}$ & Regression & $r$ & SEE, gm \\
\hline in vitro hearts & $116.5-191.8$ & 5 & $y=0.98 x+1.4$ & 0.998 & 2.5 \\
\hline
\end{tabular}

\begin{tabular}{|l|l|l|l|}
\hline \multicolumn{4}{|l|}{ Table 3. In Vitro Validation of Measurements of Mitral Annulus Size and Shape } \\
\hline Parameter & N & r squared & SEE \\
\hline Annulus area, $\mathrm{cm}^{2}$ & 10 & 0.99 & 0.9 \\
\hline Annulus circumference, cm & 10 & 0.99 & 0.3 \\
\hline Anterior leaflet area, $\mathrm{cm}^{2}$ & 9 & 0.990 & 1.0 \\
\hline Posterior leaflet area, $\mathrm{cm}^{2}$ & 9 & 0.933 & 1.1 \\
\hline $\begin{array}{l}\text { Annulus height (maximum deviation } \\
\text { from least squares fitted plane), cm }\end{array}$ & 40 & & 0.1 \\
\hline
\end{tabular}

\section{ResUlts OF VALIDATION STUdIES}

The accuracy of volume determination has been evaluated using phantoms, in vitro hearts, and in vivo by comparison with Doppler stroke volume $(1,4)$. Beaker phantoms consisted of plastic beakers whose rims were cut in a saddle shape to mimic the mitral annulus and covered with parafilm "leaflets". Balloon phantoms were imaged both in their natural ellipsoid shape and after shape distortion with an equatorial constriction. In vitro porcine and calf hearts were obtained from the slaughterhouse, stuffed, and fixed in formalin. The true volume of beaker and in vitro hearts was determined from the difference in weight when empty vs. after filling with water. In vivo Doppler stroke volume was determined by integrating flow velocity at the LV outflow track. All volumes were measured three times and averaged. Phantom and in vitro hearts were imaged in a water bath filled with saline at a concentration that mimics the speed of ultrasound in tissue at room temperature. The results indicate that our 3D echo methods are highly accurate (Table 1). Note that in the regression equation $y=$ volume calculated by $3 \mathrm{D}$ echo and $\mathrm{x}=$ true volume. 
$\mathrm{N}=$ number; $\mathrm{r}=$ correlation coefficient; $\mathrm{SEE}=$ standard error of the regression. However in vivo validation has only been performed on a small patient population to date, and additional data are needed to establish the validity of this methodology.

The accuracy of mass determination was evaluated using in vitro hearts. The porcine and calf hearts described above were trimmed, fixed, weighed in triplicate, and imaged in a saline bath (4).

The accuracy of mitral annulus and leaflet reconstruction was validated using the beaker phantoms used to validate volume measurement (3).

\section{Clinical Applications}

We feel that 3D echo offers unparalleled opportunity to explore the complex relationship between the left ventricle and mitral valve apparatus. Accordingly, we applied our 3D echo techniques to

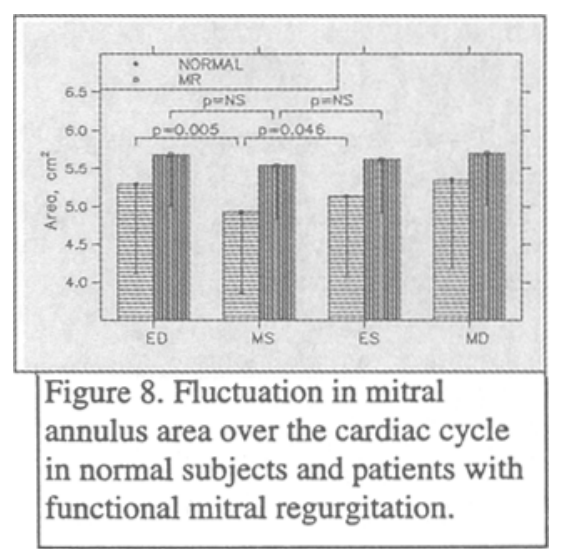

investigate the etiology of functional mitral regurgitation. This condition is characterized by a structurally normal but dysfunctional valve. It has been attributed by various investigators to distortion of the size, shape, position, or motion of the $L V$, left atrium, mitral valve annulus, and/or papillary muscles.

We analyzed the size, shape, and motion of the mitral annulus in patients with normal hearts undergoing noncardiac surgery and in patients with functional mitral regurgitation secondary to coronary artery disease undergoing bypass graft surgery and/or mitral valve repair. $3 \mathrm{D}$ echo was performed using a transesophageal echo probe following induction of anesthesia. Images were acquired at 30 frames/sec every $5-8^{\circ}$ over a $180^{\circ}$ rotation. The annulus was traced manually in every frame through a normal cardiac cycle. The traced points were converted to $\mathrm{x}, \mathrm{y}, \mathrm{z}$ coordinates and used to reconstruct the annulus with a Fourier technique (3). The analysis showed that in functional mitral regurgitation the annulus is larger, rounder, and flatter and its deformational changes during systole are reduced in magnitude, compared to normal (Fig.8) (5).

Further studies are in progress using the quantitative 3D echocardiographic techniques that we have developed to measure the size and shape of the LV and all of the elements of the mitral valve apparatus in patients with functional mitral regurgitation. This will make it possible for the first time to compare the contribution of abnormalities in each structure's shape to the severity of valvular dysfunction. Other applications of quantitative $3 \mathrm{D}$ 
echocardiography include diagnosis of ischemic heart disease by stress

echocardiography (comparing regional wall thickening at baseline and after dobutamine), endpoint measurements for clinical trials (due to the method's accuracy and reproducibility), and investigation of ventricular remodeling following acute myocardial infarction or partial left ventriculectomy.

\section{Discussion}

Earlier studies have already established the superior accuracy and reproducibility of 3D echocardiography for the measurement of LV volume, mass, and infarct size $(6,7)$.

Our methodology for 3D echocardiography is the first to provide comprehensive quantitative analysis of not only the LV but also the mitral annulus, leaflets, chordae tendineae, and papillary muscles. Other structures such as the right ventricle can also be analyzed using the same approach. The accuracy of this methodology is comparable to that of other 3D echocardiographic systems. However the advantage of our approach is flexibility, and this is the reason why it can be applied to so many more analyses than simply volume and mass measurement.

The first source of flexibility is the magnetic field system, which allows us to acquire a 3D scan using freehand scanning in the same manner as a 2D study. Images can be acquired from any combination of intersecting, parallel, or oblique imaging planes and from multiple acoustic windows, an important feature for enlarged or aneurysmal LV's and for acquiring data about other structures of interest. Because the magnetic field receiver is tiny, unlike sound emitters, it does not impinge on the mattress or interfere with apical scanning. The methods can be applied to transesophageal as well as transthoracic scanning.

The second source of flexibility is in image analysis. Borders and partial borders of any number of anatomical structures can be traced and stored. This makes it easy, for example, to measure the distance and angle between any pair of structures, or between points within the same structure.

Third, the piecewise smooth subdivision method of 3D surface reconstruction allows tremendous flexibility. Independent of geometric assumptions, it can accept borders from different combinations of views and acoustic windows. It can reconstruct any structure once the model is defined in the form of a control mesh. Mesh design is facilitated by a 3D editor allowing immediate refitting to show the effect of a design change.

Finally, we have designed the analysis software to be independent of imaging modality. That is, the same measurements can be made from magnetic resonance or computed tomography images.

In summary, our methodology allows a wider range of parameters and inter structure relationships to be analyzed than previously possible. Our plan is to apply these techniques to clinical research while at the same time researching methods for 
automating image analysis, in order to convert this into a clinical tool.

\section{REFERENCES}

1. Legget ME, Leotta DF, Bolson EL, et al. System for quantitative three dimensional echocardiography of the left ventricle based on a magnetic field position and orientation sensing system. IEEE Trans Biomed Eng 1998; (in press).

2. Bolson EL, Sheehan FH, Legget ME, et al. Applying the centersurface model to 3-D reconstructions of the left ventricle for regional function analysis. In: Computers in Cardiology. Long Beach, CA: IEEE

Computer Society, pp. 63-66 1995.

3. Legget ME, Bashein G, McDonald JA, et al. Three dimensional measurement of the mitral annulus using multiplane transesophageal echocardiography: In vitro validation and in vivo demonstration. $\mathrm{J}$ Am Soc Echo 1998;(in press).

4. Leotta DF, Munt B, Bolson EL, et al. Quantitative three-dimensional echocardiography by rapid imaging from multiple transthoracic windows: In vitro validation and initial in vivo studies. J Am Soc Echo (in press) 1997.

5. Kaplan S, Sheehan FH, Bashein G, et al. Three-dimensional echocardiographic assessment of annular dimensions, shape, and dynamics in the normal and regurgitant mitral valve (abstr.). J Am Coll Cardiol 1998;31(Suppl. A):284A.

6. Jiang L, de Prada JAV, Handschumacher $\mathrm{MD}$, et al. Quantitative three-dimensional reconstruction of aneurysmal left ventricles: In vitro and in vivo validation. Circulation 91: 222-230 1995.

\section{Gopal AS, Keller AM, Shen Z, et al.}

Three-dimensional echocardiography: In vitro and in vivo validation of left ventricular mass and comparison with conventional echocardiographic methods. J Am Coll Cardiol 24: 504-513 1994. 